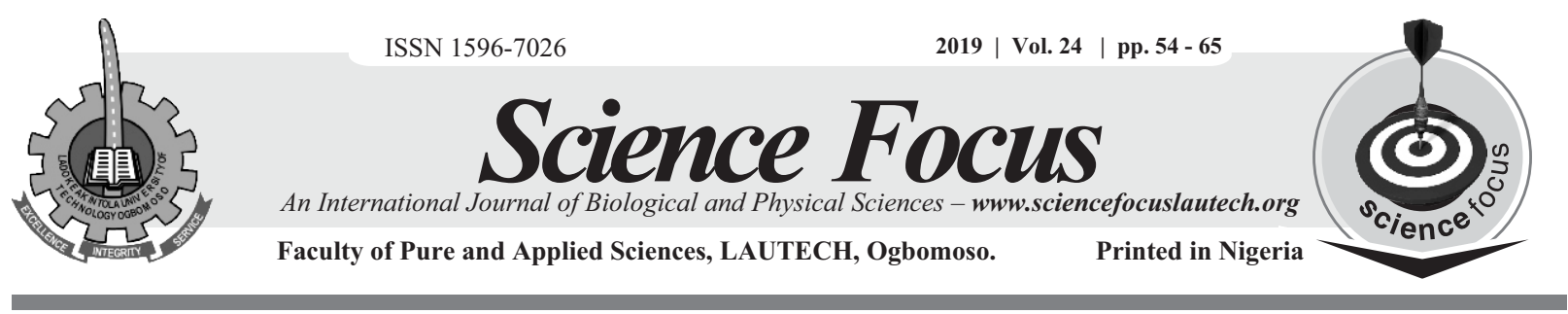

https://doi.org/10.36293/sfj.2019.0045

\title{
INTERFERENCE MITIGATION AND CONTROL MECHANISMS IN CELLULAR NETWORKS
}

\author{
Ajayi Oluwaseun Tolani, Onidare Samuel Olusayo, \\ Tiamiyu Osuolale Abdulraman, Ayeni Adeseko Aborisade \\ Department of Telecommunication Science, University of Ilorin
}

\begin{abstract}
A major problem militating against the performance of wireless cellular networks is interference. Critical performance metrics of the cellular system, such as the cell throughput, capacity, and signal quality are adversely affected by interference. Based on this understanding, we provide a realistic survey of the various interference reduction techniques in cellular networks. The fundamental causes and sources of interference in wireless cellular networks were briefly discussed before delving into the different mitigation and control mechanisms adopted for each of the different generations of cellular technology. We also discussed interference mitigation from the network and user equipment perspectives and used graphs to analyze the behavior of a Wideband Code Division Multiple Access (WCDMA) network under a cell resizing approach. This paper, in its entirety, expatiates on efficient frequency optimization and power distribution control as the fundamental interference mitigation techniques in cellular networks.
\end{abstract}

Keywords: Interference; cellular networks; frequency reuse; co-channel; LTE; $5 G$.

Corresponding Author Email:

\section{INTRODUCTION}

As mobile subscribers increase, the increase in bandwidth provision for wireless access technologies has been on the rise. Network service providers pay rapt attention to alleviating coverage and capacity problems in cellular networks, while interference and noise persist. Interference and noise, in cellular networks, have stirred the migration from one wireless access technology to another and, more importantly, interference limits the performance of cellular networks. It degrades the quality of transmission for voice and data, by introducing bit errors in the signal received. It also reduces the coverage and capacity of a network.

The First Generation of cellular networks, also known as $1 \mathrm{G}$, was introduced to provide analog voice transmission to users, using the frequency modulation technique. This technology uses frequency division multiple access (FDMA), in allocating separate channels for each mobile conversation. It employs frequency reuse in utilizing the radio spectrum in various geographical areas making the system accommodate multiple users with limited interference. One of the major challenges in this technology is noise. 
The Global System for Mobile Communications (GSM), is a second-generation technology, which uses frequency division duplex and time division multiple access (FDD/TDMA) to improve network capacity, unlike the $1 \mathrm{G}$ network, which allocates a voice frequency channel to a user. This technology allows multiple users to share a common channel of $200 \mathrm{kHz}$ which can be divided into timeslots, generated continuously for voice calls, and supports limited data capabilities (Mishra, 2004). In this generation, analog signals are converted into digital signals, before transmission.

The Universal Mobile Telecommunication System (UMTS) is a 3G technology that uses code, rather than frequency or time, in separating users. It is an improvement on the existing $2 \mathrm{G}$ network. It is aimed at improving voice quality, increasing throughput in the downlink (up to $2 \mathrm{Mbps}$ in downlink), and utilizing the frequency spectrum. The UMTS uses wideband code division multiple access (WCDMA), as the air interface technology for UMTS terrestrial radio access network (UTRAN) (Tso, et al., 2017). A factor militating against the all-round success of this technology is the interference and noise imposed by user equipment (UEs) and NodeBs.

Long Term Evolution (LTE) is an optimized allIP multimedia network, designed to provide downlink and uplink data rates, up to $300 \mathrm{Mbps}$ and $75 \mathrm{Mbps}$ respectively. The multiple access technology, employed for the downlink transmission, is orthogonal frequency division multiple access (OFDMA), while the single carrier-frequency division multiple access (SCFDMA) is used for transmissions in the uplink direction. It also uses advanced technologies such as multiple-input and multiple-output (MIMO), in improving the data rate of multimedia transmission (Tso et al., 2017).

The LTE-Advanced access network is an LTE based technology, designed to improve the capacity, coverage, and throughput of an existing LTE technology. It enhances subscribers' experience, including subscribers at the cell-edge, with higher data rate transfer, utilizing a wider bandwidth up to $100 \mathrm{MHz}$ (Onu and Alani, 2013). It is a heterogeneous network comprising of several cell sizes - micro, macro, femtocells, relays, and Home eNodeB (Onu and Alani, 2013; Kelechi et al., 2014).

The fifth-generation network also known as the $5 \mathrm{G}$ heterogeneous self-organizing and optimization network is a broadband network that uses three key features to advance broadband transmission.

i. It uses millimeter waves to transmit signals, using high frequency ranging from $30 \mathrm{GHz}$ to $300 \mathrm{GHz}$ which has not been used for mobile networks before.

ii. It uses small cells with minimal transmit power to improve user mobility and network densification in cities (cells can have a maximum coverage area of 250 meters).

iii. It uses massive MIMO which requires a base station with hundreds of ports for antennas in transmitting and receiving signals (Nordrum, et al., 2017).

The $2.5 \mathrm{G}, 3 \mathrm{G}$, and $4 \mathrm{G}$ technologies are used independently, and hence, no combination of different wireless technologies for the same session (such as VoIP and FTP). The 5G technology allows mobile terminals to use errorcontrol schemes, software-defined radios, and modulation schemes that enable simultaneous access to multiple wireless technologies (Gohil, et al., 2013). This technology also employs the use of beamforming and full-duplex to determine the most efficient route to a user and double the capacity of the network respectively (Nordrum, et al., 2017).

\section{Causes of Interference in Cellular Networks}

In general, different systems and services such as mobile communications, digital video broadcasting, wireless local area networks, and mobile radios are, each, assigned a specific spectrum, to avoid the anomaly of having different services, transmitting on the same frequency, thereby causing interference (Viavi, 2015). Also, other than interference due to radio frequency (RF) leakages, harmonics, or frequency drifts, cell sites are subject to interferences generated from improper connectivity of RF devices such as antennas, connectors, and cables (Viavi, 2015). 
Two types of interference are considered in cellular networks - co-channel interference and adjacent channel interference. In cellular networks, the former (i.e. co-channel) is interference due to frequency reuse. Factors such as reuse distance and carrier transmit power influence co-channel interference between cells. Adjacent channel interference, on the other hand, is a consequence of signals from neighboring frequencies interfering with the desired signal of another channel (Heiska, 2003). Imperfect receiver filters and improper channel assignment influences adjacent channel interference.

GSM networks use frequency allocation methods for base stations to transmit and receive signals from mobile stations (MS). These frequencies are reused at different cells. Reusing the same frequency between base stations with relatively short distances from each other, pose the problem of co-channel interference on the network, just as using adjacent frequencies for base stations on the network pose adjacent interference. Hence, for effective interference mitigation in GSM, factors such as the transmitter-receiver distance, azimuth, frequency reuse, and location of the network should be given adequate consideration. (Lan, et al., 2011). The WCDMA technology, used in 3G like its $2 \mathrm{G}$ predecessor, the CDMA, enables concurrent sharing of, and transmission on, the same RF carrier by different users (Mohan and Ravichandran, 2012). Some specialized codes known as "channelization codes" are added to the transmission, over the UMTS air interface to differentiate signals from different UEs. The interference level is dependent on the user density in a particular cell, which is a function of the cell radius and capacity of the network (Mohan and Ravichandran, 2012). The higher the number of users on the network, the higher the interference level and the smaller the cell radius (Masmoudi and Tabbane, 2006). For a WCDMA network, the major causes of interference are - in-cell traffic, thermal noise, other cell traffic and external traffic (Abdelouahed,

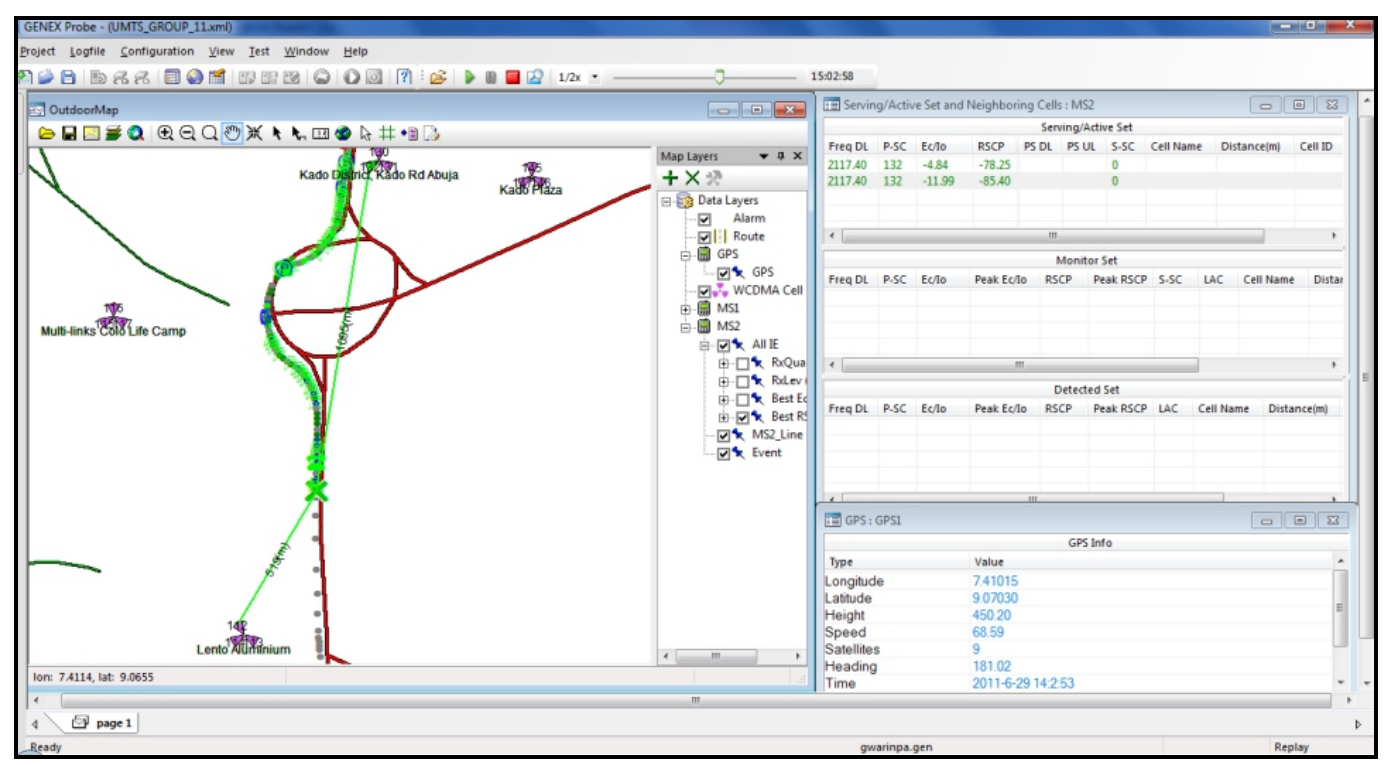

Fig. 1: High interference in WCDMA

According to Kelechi et al. (2014), as LTE networks aim at improving network capacity, by efficiently utilizing wideband frequency, interference is a major problem militating against its success as systems compete for finite amounts of spectrum. However, interference in LTE is unpredictable and depends on the channel condition of the system, traffic from other terminals as well as noise. Network operators have invested in constructing multiple base towers and filters to control interference in LTE but increase in the number of subscribers on the spectrum has made it difficult to keep the technology free from interference (Kelechi et al., 2014). Dahlman (2007) and Sesia (2009), affirm that LTE OFDMA is used to ensure subcarriers orthogonality, thereby eliminating intra-cell interference. However, inter-cell 
interference in LTE degrades system performance metrics such as spectral efficiency and throughput, particularly for users at the edge of the cell. Therefore, interference mitigation and control mechanism in LTE is necessary for optimal and acceptable system performance.

The LTE-Advanced is still not an interferencefree heterogeneous network technology as it integrates multiple femtocells and picocells into the macro cell. A major cause of interference in LTE-Advanced is the power difference between nodes (Kelechi et al., 2014). Network operators deploy femtocells and picocells to increase coverage and data throughput, but this strategy still poses a great deal of interference problem, on the network, as users tend to connect to the macro cell (with high transmit power) rather than the picocell or femtocell that has low path loss. Hence, users connected to the macro cell will interfere with low power nodes in the same location (Kelechi et al., 2014).

As shown in Figure 2, the $5 \mathrm{G}$ heterogeneous network consists of a macro base station (MBS) and under-laid full-duplex (FD-enabled) small cells (SCs). The MBS uses massive MIMO antennas to serve macro UEs and provide wireless backhaul system to neighboring FDenabled SCs. Each FD-enabled SC connected to the MBS serves its UEs and other macro UEs attached to it at the same frequency band. The SCs use FD to double the network capacity. The FD-enabled SC cause both co-tier and cross-tier unwanted interference with the macro UE and other UEs (Vu, et al., 2017).

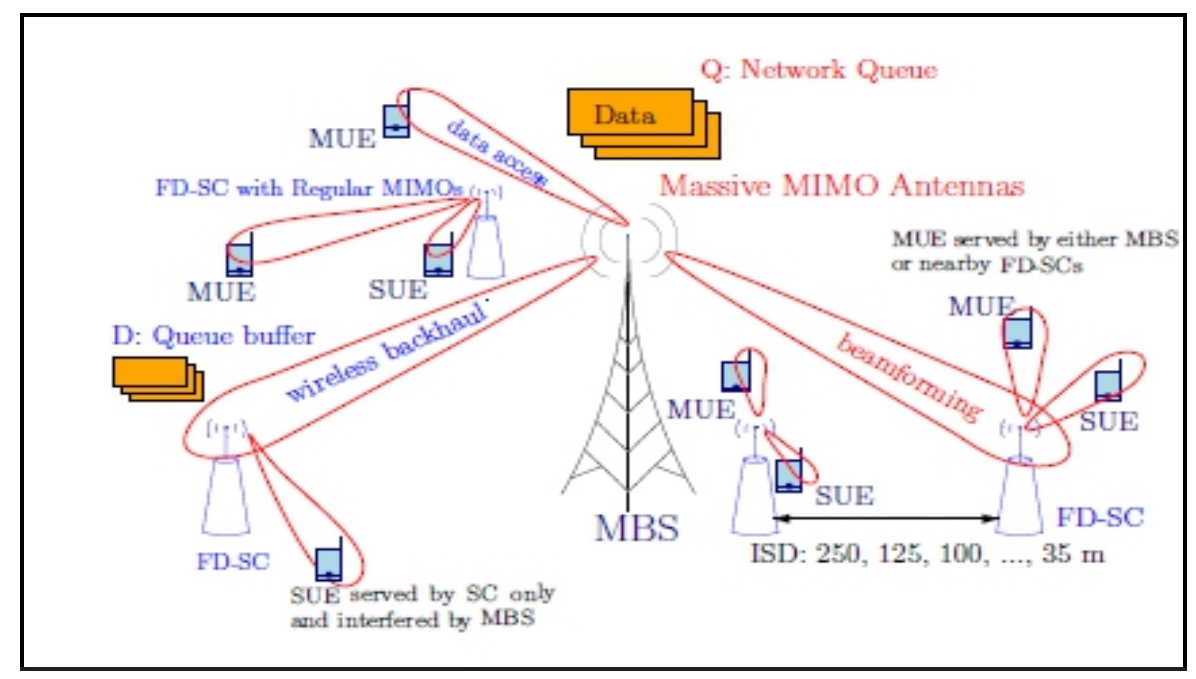

Fig. 2: Network Scenario in 5G (Vu, et al., 2016)

\section{GUARD BAND IN 1G}

In the first generation of cellular networks, users were separated by frequencies and each frequency band had a guard band/channel spacing of $30 \mathrm{kHz}$ (for Advanced Mobile Phone
System). This guard band is required to control and mitigate adjacent channel interference between users and cells. Usually, mobile terminals have one frequency band both in uplink and downlink (Tso, et al., 2017).

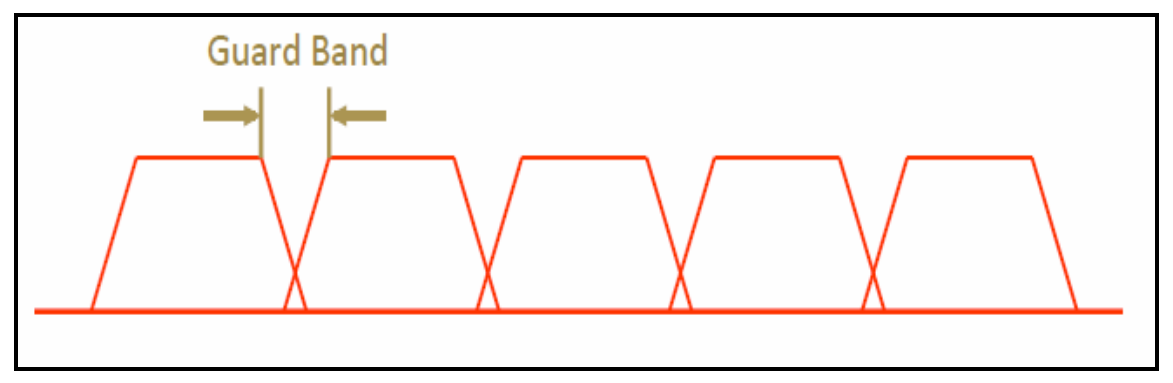


Fig. 3: Guard band in 1G network (Tso, et al., 2017)

\section{POWER CONTROL AND FREQUENCY OPTIMIZATION IN GSM}

\section{Optimum Power Distribution}

The quest to maximize the available traffic channels in a geographical area result in cochannel and adjacent channel interference. Therefore, the fundamental interference mitigation technique used in GSM to reduce cochannel interference is controlling the transmit power of base stations transmitting in a relatively small region. With power control, the power of each transmitter is adjusted for a given channel allocation which minimizes interference. Optimum transmitted power distribution method is appropriate for planning the energy balance of a cellular system (Sumithabhashini and Reddy, 2008).

Max Power $P_{\max }=\left(P_{1 \max }, P_{2 \max \ldots} . P_{\text {Nmax }}\right)$

where $P_{1 \max } P_{2 \max \ldots .} P_{N \max }$ each represents the transmit power of the base station (BS) transceivers for each cell.

Lowering the antenna height is another effective way of reducing co-channel interference in GSM especially in a flat terrain or valley situation. For a hill, the effective height of an antenna is given as

$h_{e 1}=h_{1}+H$

where ${ }^{H}$ is the height of the hill and $h_{1}$ is the height of an antenna. Lowering $h_{l}$ to half of its initial value is given as

$h_{\text {new }}=h_{1} / 2$

Hence, the effective height of the antenna after lowering $h_{l}$ is

$$
h_{e 2}=0.5 h_{1}+H
$$

$$
G=20 \log 10\left[\frac{h e 2}{h e 1}\right]=G=20 \log 10\left[1-\frac{0.5 h 1}{h 1+H}\right]
$$

This reduction in antenna height reduces cochannel interference between cell sites as cells are prevented from overshooting into neighboring coverage zones.

\section{Frequency Optimization Algorithm}

More than anything else, the cause of co-channel and/or adjacent channel interference is the site layer (shortest path) between cells and the frequency allocation in cells. Therefore, cell coverage, frequency allocation, and site layers are parameters that should be checked when mitigating interference in GSM. A frequency optimization algorithm is used to mitigate and control interference in GSM systems (Lan, et al., 2011). Co-channel and/or adjacent channel interference can be identified based on whether or not they are on the sine cell, adjoining cell, or having azimuthal sparring. Furthermore, interference levels are quantized into eight (8) levels (level 0 to level 7) from low interference to high interference.

A frequency interference vector that analyses the cell site layers and interference level of cells can be defined as $\boldsymbol{B}=\left(b_{1}, b_{2}, b_{3}, b_{4}, b_{5}, b_{6}\right)$ where $b_{1}$ is the main interference level, $b_{2}$ is the smallest (nearest) site layer of the main interference source, $b_{3}$ represents the number of the main interference sources, $b_{4}$ represents the smallest site layer of the intefference source, $b_{5}$ represents the number of interference sources in the smallest site layer $b_{4}$; and $b_{6}$ represents the total number of interference sources (Lan, et al., 2011). This is also referred to as the performance vector for each frequency of a cell. For example, consider 2 frequencies of a cell with the following performance vectors:

$$
\begin{aligned}
& \boldsymbol{B}_{1}=(4,2,1,2,1,4) \\
& \boldsymbol{B}_{2}=(4,4,1,2,2,5)
\end{aligned}
$$

The gain reduction is 
From the frequency performance vectors given above, $\boldsymbol{B}_{\mathbf{1}}$ and $\boldsymbol{B}_{\mathbf{2}}$ have the same interference level $b_{1}$ of 4 , the site layer $b_{2}$ of the main interference source for $\boldsymbol{B}_{\mathbf{1}}$ and $\boldsymbol{B}_{\mathbf{2}}$ are 2 and 4 respectively. This implies that the site layer with the main interference source is farther away in $\boldsymbol{B}_{\mathbf{2}}$ than $\boldsymbol{B}_{\mathbf{1}}$. The element $b_{3}$ of $\boldsymbol{B}_{\mathbf{1}}$ and $\boldsymbol{B}_{\mathbf{2}}$, both have the same number of main interference sources. Therefore, the interference level is $\boldsymbol{B}_{\mathbf{1}}>$ $\boldsymbol{B}_{\mathbf{2}}$ and the best frequency is $\boldsymbol{B}_{\mathbf{2}}$.

The interference vector can be used to evaluate the level of interference existing at different frequencies. Thus, frequency optimization is achieved by replacing the frequencies suffering high interference with the best frequencies. A six-step frequency optimization algorithm is established thus:

1. Calculate the site layers of BSs in the network.

2. Determine the frequency interference vector for each frequency $i$ of each cell in the network $\boldsymbol{B}_{\boldsymbol{i}}$.

3. According to $\boldsymbol{B}_{i}$, arrange the cells' frequencies, determine the cell(s) with the largest interference level and sort the cells into the list of cells ' $\mathrm{C}$ ' to be optimized.

4. Evaluate the frequency in the frequency set and select the best frequency to replace the interfering frequency for better frequency optimization.

5. End the optimization process if cells in ' $\mathrm{C}$ ' are empty, no change in the frequency, or the optimization process reaches its maximum, else, return to step 2.

6. Output the result if the frequency optimization objective is fulfilled.

\section{WCDMA MULTIPLE ACCESS INTERFERENCE CANCELLATION}

In WCDMA, the direct-sequence code-division multiple access system, is effective against cochannel interference, as users are mutually orthogonal. However, the orthogonality of the codes is not preserved because the increased bandwidth is equivalent to a smaller chip period and spreading factor. This, in turn, leads to increased multipath resolution/propagation, thus, increasing interference within the cell (Liu and Zarez, 2007). A multiple access interference (MAI) cancellation technique is described by comparing the performance of RAKE receivers and Equalizers in the successive cancellation of interfering multipath signals from the received signal. The RAKE receiver technique provides an acceptable performance when there are few active users and requires less complex signal processing, however, as users increase, the nonzero correlation between spreading sequences become pertinent, thereby degrading the performance of RAKE receivers. On the other hand, the use of an equalizer enhances the downlink performance for receivers but suffers from high complexity (Liu and Zrez, 2007; Choi, et al., 2004; Ueng, et al., 2006).

The following scenario illustrates the technique for MAI cancellation using computer simulation.

\section{Illustration 1:}

The downlink of a WCDMA system with a chip of 3.84Mcps, a saturated load of $K=64$ active users, and orthogonal Walsh codes of length 64 for users' spreading codes were considered, alongside a common random scrambling code. The baseband signal transmitted for the $K_{t h}$ user is deduced by:

$x_{k}(t)=A_{k} \sum_{m=0}^{M-1} b_{k}(m) s_{k}(t-m T), \quad k=1,2, \ldots, K$

where $M, T, A_{k}$ and $S_{k}(t) s$ are the number of data symbols per frame, symbol interval, amplitude, and normalized signature waveform for the $K_{t h}$ user respectively.

Four multipath propagation channels were assumed - one at zero propagation delay and others at propagation delays randomly selected within the chip time interval $\left[T_{c}, 25 T_{c}\right]$. These propagation delays are feasible for outdoor propagation environment with the maximum spreading delay spanning up to $7 \mu$ s (Liu and 
Zarez, 2007). The channel paths defined in (Liu and Zarez, 2007), were assumed to have powers of $0 \mathrm{~dB},-3 \mathrm{~dB},-13 \mathrm{~dB}$ and $-25 \mathrm{~dB}$ as well as path phases between 0 and $2 \pi$. This allows the receivers to cancel the signals from interfering paths using the chips alongside knowledge of channel parameters. The results of the simulation were obtained for a frame length of 500 bits by averaging over 300 simulation runs. It revealed that the bit error rate (BER) performance using the technique presented in (Liu and Zarez, 2007) outperforms the RAKE receiver and single-user system for different SNR $\left(E_{b}, N_{0}\right)$, but it has the loss of few dBs which resulted from the accumulated noise. Conclusively, the only disadvantage of this technique is the inherent noise accumulated, which can affect the performance of the receiver (Liu and Zarez, 2007).

\section{WCDMA CELL RESIZING APPROACH}

In this technique, the signal-to-noise ratio (SNR) value of each cell is measured, using an access point (AP) (Mohan and Ravichandran, 2012). The AP based on the measured SNR value is classified into three categories namely: normal, saturated, and cooperative. A cell is normal if the SNR value is equal to the average SNR value. Cells with SNR values greater than the average SNR value are the saturated cells. Cells with SNR values below the average SNR value are the corporative cells. When an AP finds the SNR greater than the average SNR, in a particular cell, it invokes cell-resizing approach. A SUPPORT-REQ message is transmitted from a saturated cell to all its neighboring cells. Upon reception of the message, the cooperative cells reply with a SUPPORT-REP message. If the SUPPORT-REP message received by the saturated cell is more than one, it identifies the cell that is closer to it. Consequently, the saturated cell reduces its size thereby expelling cell-edge users. At the same time, the cooperative cell enlarges in size to accept users expelled from the saturated cells. The cell users balancing adopted by the cell resizing concept mitigates interference in WCDMA networks (Mohan and Ravichandran, 2012).
Figures 4 and 6 show the cell resizing approach in WCDMA and its graphical representation respectively.

\section{Illustration 2:}

As shown in Figure 4, cell $\mathrm{A}$ is preloaded with 7 UEs thereby increasing the cell noise rise level in the downlink and interference between UEs in the uplink (each user has a throughput of 285 $\mathrm{Kbps}$ ). Cell $\mathrm{B}$ is preloaded with 4 UEs making each user throughput 500 Kbps. An implementation of the WCDMA cell resizing approach balances the throughput of both cells (Cell $\mathrm{A}=333 \mathrm{Kbps}$ and Cell $\mathrm{B}=400 \mathrm{Kbps})$.

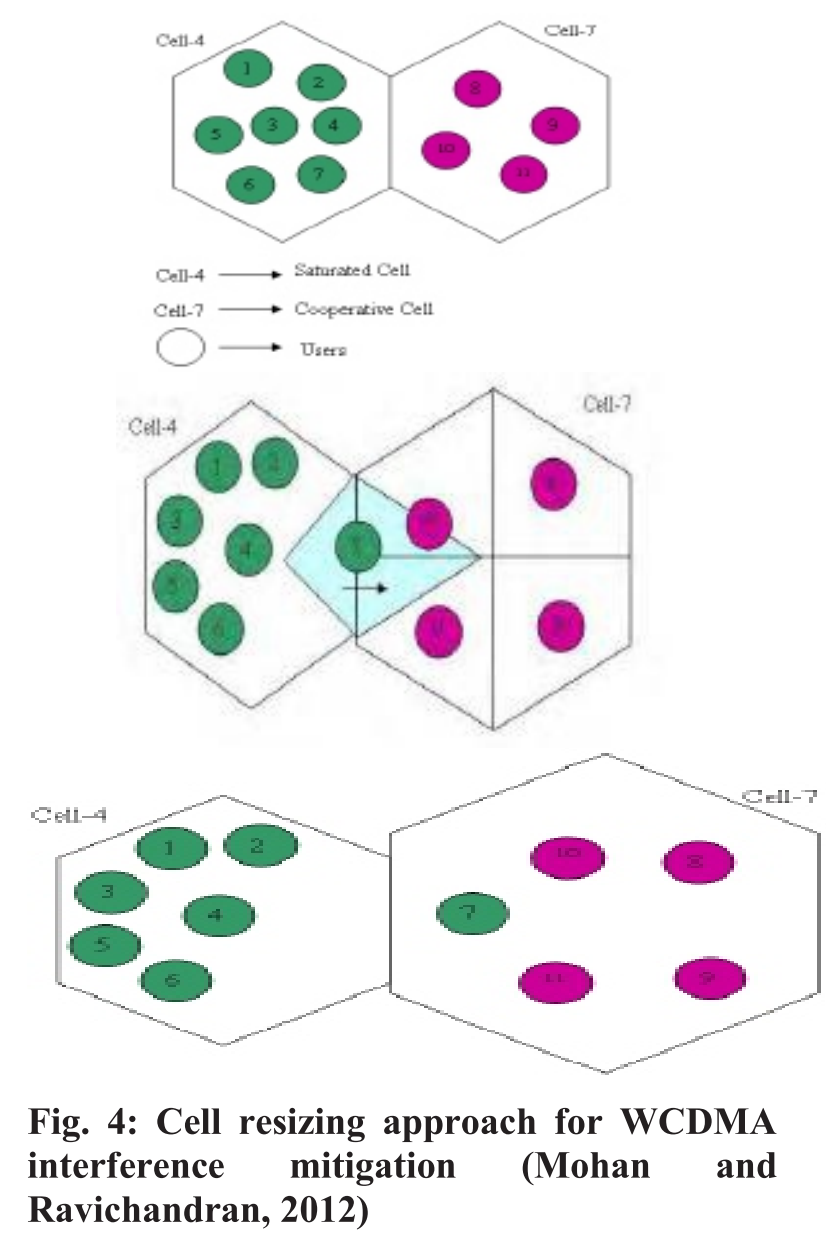




\begin{tabular}{|c|c|c|c|c|}
\hline \multicolumn{5}{|c|}{ CELL RESIZING IN WCDMA } \\
\hline Time & Cell A (kbps) & Cell B (kbps) & \\
\hline $0: 00$ & 285 & 500 & Cell A =7, Cell B = 4 \\
\hline $0: 50$ & 285 & 500 & \\
\hline $1: 00$ & 285 & 500 & \\
\hline $1: 50$ & 285 & 500 & Cell A = 6, Cell B=5 \\
\hline $2: 00$ & 333 & 400 & \\
\hline $2: 50$ & 333 & 400 & \\
\hline $3: 00$ & 333 & 400 & & \\
\hline $3: 50$ & 333 & 400 & \\
\hline
\end{tabular}

Fig. 5: Cell Resizing with effect on Throughput in WCDMA

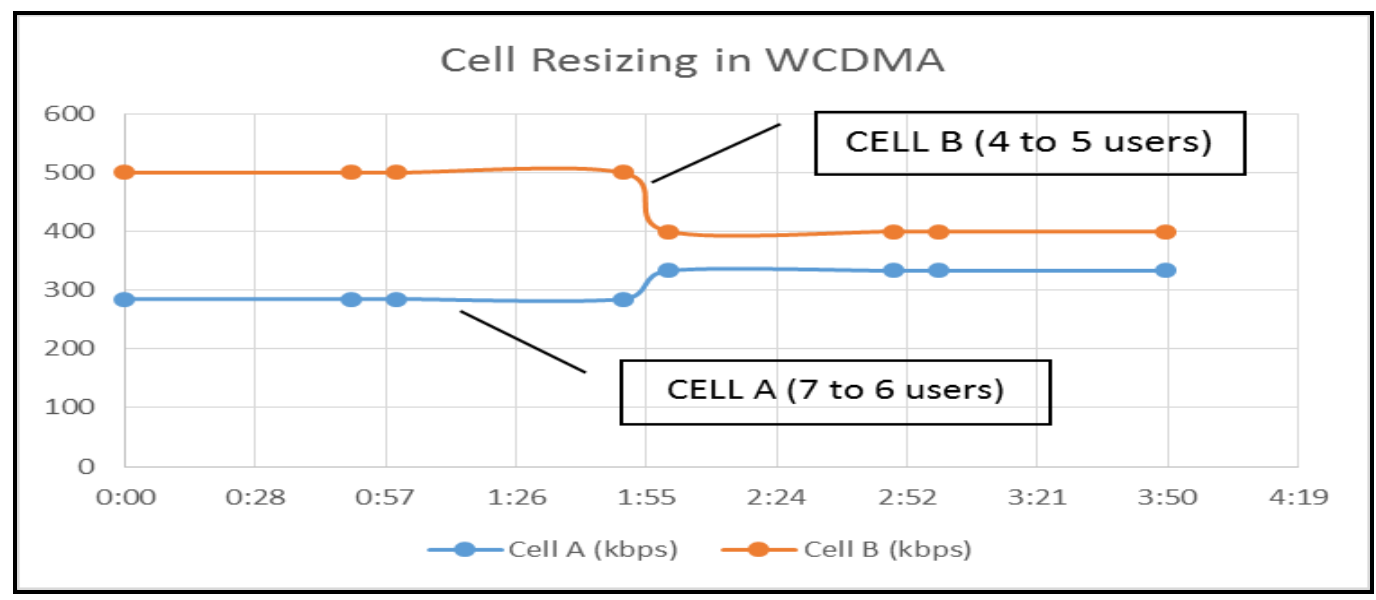

Fig. 6: Graphical representation of cell resize approach

\section{INTERFERENCE MANAGEMENT IN LTE}

\section{Interference Avoidance and Randomization}

One of the major problems militating against the success of LTE networks is the dense reuse of frequencies in cells, geared towards improving network capacity. This, however, poses interference which can be intra-cell and/or intercell interference (ICI). This interference, degrades the LTE's throughput and spectral efficiency, particularly for users at the cell-edge. When a user moves from a serving eNodeB and approaches an adjacent $\mathrm{eNodeB}$, the received signal to interference noise ratio (SINR) decays as the received signal reference power (RSRP) decreases and ICI increases. Two schemes interference avoidance and interference randomization are identified for interference management in LTE downlink networks (Afroz, et al., 2015). Firstly, the interference avoidance scheme controls various radio resources (frequency, time, and power) to increase SINR and throughput for cell-edge users, without sacrificing the throughput of cell center users. Based on the timescale, the interference avoidance scheme can be classified as static, semi-static, and dynamic. The static scheme operates on a relatively large timescale (Saquib, et al., 2013; Hussain, 2009). During the radio network planning stage, the static interference avoidance scheme determines the allocation of radio resources for each cell while long-term readjustments are made as demanded by prevailing conditions during the network operation. Therefore, the power levels and subcarrier sets are fixed (static) for each cell and cell region.

The semi-static scheme and dynamic scheme differ from the static scheme with respect to the timescale of resource allocation (Bosisio and Spagnolini, 2008). While the semi-static scheme 
focuses on allocating radio resources dynamically for cell-edge users, in a dynamic scheme, resource allocations are done at very short periods. Secondly, in the interference randomization scheme, the users' data in each cell is spread across a time-frequency channel and subcarriers, thereby randomizing interference scenarios and achieving frequency diversity gain. The subcarriers permutate transmission in a pseudorandom manner to ensure the spreading of interference along with the transmission of a particular user. Afroz, et al. (2015) and Bosisio (2008), agree that the scheme is suitable for a practical system as there is no signaling overhead and less complex resource management. Therefore, the interference avoidance schemes put restrictions on the downlink resource usage (frequency, time, and power). These restrictions help mitigate the interference generated in mobile cellular networks such as the downlink LTE system.

\section{Partial Frequency Reuse}

To mitigate interference in LTE networks, some inter-cell interference mitigation and control mechanisms are implemented during signal transmission or reception. The concept of allocating a specific portion of the LTE bandwidth to cell-edge and cell center users is an approach in mitigating interference in LTE.

Partial Frequency Reuse is an advancement on the conventional frequency reuse, in which the entire spectrum band is divided into four subbands using reuse factor 1 (RF1) for cell center users and RF3 for cell-edge users. This improves the SINR of cell-edge users as inter-cell interference is reduced by this scheme (Khan, 2009). Partial frequency reuse does not, efficiently, utilize the bandwidth, thereby leading to lower cell throughput. It aims at reducing interference at the cell-edge rather than improving cell throughput.

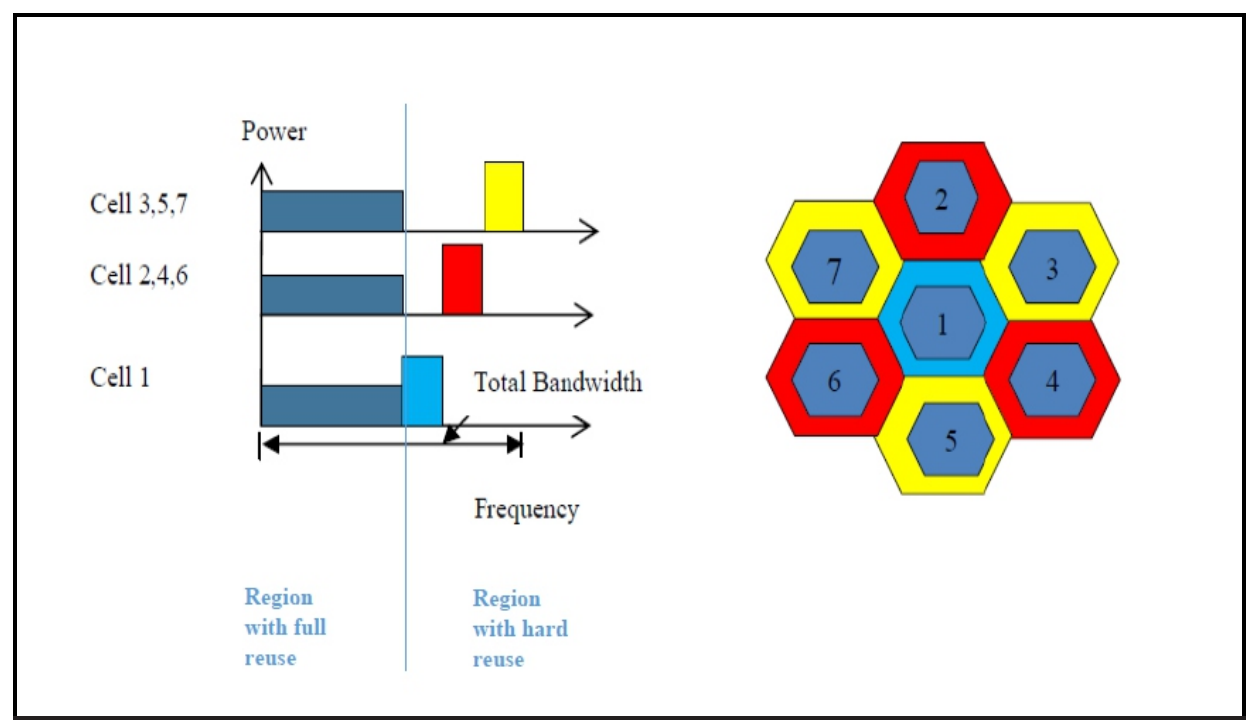

Fig. 7: Frequency planning for PFR scheme (Afroz, et al., 2015)

\section{ENHANCED ICIC TECHNIQUE FOR LTE- ADVANCED}

Inter-cell interference coordination (ICIC) technique specified in LTE (Release 8 and Release 9) may not be effective in mitigating interference in LTE-Advanced, therefore a proposal for enhanced ICIC was developed for LTE-Advanced (Kelechi, et al., 2014). This technique is grouped into three categories: i. Time Domain Technique: In this technique, multiple user equipment in a femtocell can be scheduled, based on time within the subframes of an overlapping femtocell to mitigate crosstier interference.

ii. Frequency Domain Technique: In this enhanced ICIC technique, the control channels and physical signals of different cells are scheduled, based on 
bandwidth reduction, to improve the orthogonality of signal transmission between different cells.

iii. Power Control Technique: This is a more effective method of reducing the potential tendency of interference in LTE-Advanced by reducing the RF power generated by femtocells. Power control technique solution reduces the throughput of the femtocell but protects users at the cell edge from excessive interference.

\section{INTERFERENCE MITIGATION IN 5G}

\section{Advanced Interference Technique \\ Management}

The network-side interference management used in $4 \mathrm{G}$ LTE is beneficial as it is backward compatible with legacy users and systems (Nam, et al., 2014). However, sole reliance on the network to mitigate interference is a major setback for the LTE network as it poses significant backhaul and feedback overhead problems. The user equipment (UE)-side interference management has been identified to be a solution in alleviating the network side interference management issue (Bandemer, et al., 2012; Baccelli, et al., 2011), though it can be synergized with the network side interference management (Akdeniz and Rangan, 2013; Abdrashitov, et al., 2014). Sequel to the findings from recent studies on the LTE network, Nam, et al. (2014) attests that introducing the advanced interference management (AIM) technique, which consists of the UE-side and network side technique would be a key driver to the $5 \mathrm{G}$ network. Two aspects of the AIM techniques were identified:

i. UE-side interference management by advanced receivers with interference joint detection/decoding.

ii. Network-side interference management by joint scheduling.

The first aspect of the AIM technique advanced receivers, has the capability to take advantage of the coding scheme, modulation technique, channel, and resource allocation of the interference signal. The advanced receiver detects the symbols of the interference signal and tries to decode them, so they can be reconstructed and cancelled from the received signal to improve the performance of the desired signal. The second aspect of the AIM technique - joint scheduling, focuses on improving the performance at the cell-edges through coordinated transmission (called coordinated multiple point) among multiple transmitters at different cell sites. In addition, the combination of advanced receivers and joint scheduling provides cell-edge throughput up to $50 \%$. The adoption of AIM for the 5G network will provide an optimally edgeless end-user experience.

\section{Joint Load Balancing for 5G Heterogeneous Network}

Interference in $5 \mathrm{G}$ heterogeneous network can be reduced by setting the macro BS to handle macro UEs queue while the SCs handle small cell UEs data (Vu, et al., 2016). The SC can balance the load on the macro BS based on the wireless backhaul link requirement. In any situation where the link between the macro UE and macro BS is better than that of the link between the macro UE and $\mathrm{SC}$, then the macro BS should serve the macro UE rather than being served by the SC. The macro BS is designed with antennas to serve macro UEs and SCs while other antennas are used to mitigate co-tier or cross-tier interference.

\section{CONCLUSION}

The success of cellular networks is not only dependent on the increase in capacity, but also on the amount of suppressible interference and noise which regularly pose signal distortion problems. This paper provides a high-level overview of the control mechanisms and techniques viable in mitigating interference in cellular networks: 1G, GSM, WCDMA, LTE, LTE-Advanced, and $5 \mathrm{G}$ heterogeneous. We discussed interference mitigation from the network and UE perspectives, as well as used graphs to analyze the behavior of a WCDMA network under a cell resizing approach. In general, transmission power distribution between cell sites and frequency optimization 
are the most realistic and fundamental methods of interference reduction in cellular networks.

\section{References}

Abdelouahed, J. (2012). A Theoretical Prediction of Users in WCDMA Interference. In Journal of Theoretical and Applied Information Technology, vol. 35, no. 2, pp. 164-165.

Abdrashitov, V., Nam, W., and Bai, D. (2014, May). Rate and UE Selection Algorithms for Interference-Aware Receivers. In Proceedings of IEEE Vehicular Technology Conference, VTC2014-Spring, Seoul, pp. 1-5.

Afroz, F., and Sandrasegaran, K. (2015). Interference Management in LTE Downlink Networks. In International Journal of Wireless \& Mobile Networks (IJWMN), vol. 7, no. 1, pp. 91-96.

Akdeniz, M. R., and Rangan, S. (2013, July). Optimal Wireless Scheduling with Interference Cancellation. In Proceedings of IEEE International Symppsium on Information Theory, Istanbul, Turkey, pp. 246-250.

Baccelli, F., El Gamal, A., and David, N. C. (2011, May). Interference Networks with Pointto-Point Codes. In IEEE Transactions on Information. Theory, vol. 57, no. 5, pp. 25822596.

Bandemer, B., El Gamal, A., and Kim, Y. H. (2012, October). Simultaneous non-unique Decoding Is Rate-Optimal. In IEEE 2012 50th Annual Allerton Conference on Communication, Control, and Computing (Allerton), pp. 9-16.

Bosisio, R. and Spagnolini, U. (2008, April). Interference Coordination vs. Interference Randomization in Multicell 3GPP LTE System. In IEEE Wireless Communications and Networking Conference, pp.824-829.

Choi, J., Kim, S. R., and Lim, C. C. (2004). Receivers with chip-level decision feedback equalizer for CDMA downlink channels. In IEEE Transactions on Wireless Communications, 3(1), pp. 300-314.
Dahlman, E., Parkvall, S., Skold, J., and Beming, P. (2010). $3 \mathrm{G}$ evolution: HSPA and LTE for mobile broadband, second ed. Academic press.

Gohil, A., Modi, H., and Patel, S. K. (2013, March). 5G technology of mobile communication: A survey. In 2013international conference on intelligent systems and signal processing (ISSP) (pp. 288-292). IEEE.

Heiska, K. (2003). Interference between GSM/EDGE and Other Cellular Radio Technologies, second ed. John Wiley \& Sons Ltd.

Hussain, S. (2009). Dynamic Radio Resource Management in 3GPP LTE (Master's thesis, Blekinge Institute of Technology). Retrieved from

https://pdfs.semanticscholar.org/d2c6/a18f268e 1 fd8ef53fcd6b54f8fc5bb38824c.pdf

Interference in Cellular Networks: Intermodulation and Frequency Refarming. Viavi Solutions Inc. https://www.viavisolutions.com/enus/literature/interference-cellular-networksintermodulation-and frequency-refarming-whitepaper-en.pdf (accessed 26 May 2017).

Kelechi, I. G., Uchendu, O. E., Tochukwu, N. E., Ikechukwu, N. K., and Ikenna, U. A. (2014). Inter-Cell Interference Mitigation Techniques in a Heterogeneous LTE-Advanced Access Network. In International Journal of Advanced Engineering and Nano Technology (IJAENT), 1(12), pp. 9-15.

Khan, F. (2009). LTE for 4G Mobile Broadband: Air Interface Technologies and Performance, first ed. Cambridge University press.

Lan, L., Gou, X., Mao, J., and Ke, W. (2011). GSM co-channel and adjacent channel interference analysis and optimization. Tsinghua Science and Technology, 16(6), 583-588. 
Liu, D., and Zare, H. (2007). A multipath interference cancellation technique for WCDMA downlink receivers. International Journal of Communication Systems, 20(6), 661-668.

Masmoudi, A. and Tabbane, S. (2006). OtherCell-Interference factor Distribution Model in Downlink. ACM International Journal of Wireless Personal Communications, vol. 36, no. 3, pp. 245-275.

Mishra, A. R. (2004). Fundamentals of Cellular NetworkPlanning and Optimization $2 \mathrm{G} / 2.5 \mathrm{G} / 3 \mathrm{G}$...Evolution to $4 \mathrm{G}$, first ed. West Sussex England: John Wiley \& Sons Ltd.

Mohan, N. and Ravichandran, T. (2012). Interference Reduction Technique in WCDMA using Cell Resizing. International Journal on Computer Science and Engineering (IJCSE), vol. 4, no. 7, pp. 1330-1334.

Nam, W., Bai, D., Lee, J., and Kang, I. (2014). Advanced interference management for $5 \mathrm{G}$ cellular networks. In IEEE Communications Magazine, 52(5), 52-60.

Nordrum, A., Clark, K., and IEEE Spectrum Staff. (2017, January). Everything you need to know about 5G.https://spectrum.ieee.org/video/telecom/wirel ess/everything-you-need-to-know-about-5g (accessed 15 June 2017).

Onu, E., and Alani, O. (2013, June). LTEAdvanced and Beyond. In 14th Annual Post Graduate Symposium on the Coverage of Telecommunications, Networking and
Broadcasting, Liverpool, United Kingdom, pp. 1-5.

Saquib, N., Hossain, E., and Kim, D. I. (2013). Fractional frequency reuse for interference management in LTE advanced hetnets. IEEE Wireless Communications, 20(2), 113-122.

Sesia, S., Toufik, I., and Baker, M. (2011). LTEthe UMTS long term evolution: from theory to practice, second ed. John Wiley \& Sons.

Sumithabhashini, P., and Reddy, N. E. (2008). Comparison of Various Techniques for Suppression or Cancellation of Co Channel Interference in GSM. Medwell Journal of Mobile Communication, 2(3), 73-77.

Tso, P., Vishwanath, H., Eran L., Lorenco, J., and Velrajan, S. (2017, February). Mobile Handset Cellular Network. https://www.slideshare.net/Raafatyounis/5432cellular-network. (accessed 15 June 2017).

Ueng, F. B., Wang, H. F., Chang, R., and Jeng, L. D. (2006, December). Zero forcing and minimum mean square error equalization for OFDM-CDMA multiuser detection in multipath fading channels. In 2006 International Symposium on Intelligent Signal Processing and Communications (pp. 505-509). IEEE.

Vu, T. K., Bennis, M., Samarakoon, S., Debbah, M., \& Latva-Aho, M. (2017). Joint load balancing and interference mitigation in $5 \mathrm{G}$ heterogeneous networks. IEEE Transactions on Wireless Communications, 16(9), 6032-6046. 